\title{
Left Ventricular Mid Inferoseptal Segment
}

National Cancer Institute

\section{Source}

National Cancer Institute. Left Ventricular Mid Inferoseptal Segment. NCI Thesaurus.

Code 127666.

The inferoseptal portion of the mid-cavity division of the left ventricular myocardium as determined using the AHA 17-Segment Model (Cerqueira et al., 2002). 